\title{
Duración de la supresión viral en pacientes con VIH en terapia antiretroviral en Perú: análisis de sobrevida acumulada 2004-2012
}

\author{
Duration of viral suppression in HIV patients on antiretroviral therapy in Peru: \\ cumulative survival analysis 2004- 2012
}

\author{
Patricia Caballero Ñopo ${ }^{1,2, a}$, Jorge Alarcón Villaverde ${ }^{1,3, b}$, Julia Rosa Piscoya Sara ${ }^{1,3, c}$, Nora Reyes \\ Puma $^{1,2, d,}$ Soledad Romero Ruiz ${ }^{4, e}$, Cristian Obregón Cahuayo ${ }^{2, f,}$ Gustavo Alonso Urbano Quispe ${ }^{5}$ \\ Instituto de Medicina Tropical Daniel A. Carrión, Facultad de Medicina, Universidad Nacional Mayor de San Marcos. Lima, Perú. \\ 2 Unidad de Análisis y Generación de Evidencias en Salud Pública, Instituto Nacional de Salud, Centro Colaborador Cochrane. Lima, Perú. \\ ${ }^{3}$ Centro de Investigaciones Tecnológicas, Biomédicas y Medioambientales. Lima, Perú. \\ ${ }^{4}$ Laboratorio de Referencia Nacional de VIH, Instituto Nacional de Salud. Lima, Perú. \\ 5 Estudiante de medicina, Facultad de Medicina, Universidad Nacional Mayor de San Marcos. Lima, Perú. \\ a Médico cirujano, Maestro en Ciencias en Salud Pública \\ b Médico cirujano, Doctor en Medicina \\ - Médico cirujano, Maestro en Ciencias \\ 'Médico cirujano, Maestro en Salud Pública \\ • Bióloga-microbióloga, Magister en Salud Pública \\ Licenciado en estadística
}

An Fac med. 2018; 79(2):125-30 / http://dx.doi.org/10.15381/anales.v79i2.14938

\author{
Correspondencia: \\ Patricia Caballero \\ Dirección: Ciudad Universitaria. \\ Instituto de Medicina Tropical "Daniel \\ A. Carrión". Sección Virología \\ Teléfono: 6197000 anexo 4404 \\ Correo electrónico: zcaballeron@ \\ unmsm.edu.pe
}

Recibido: 29 de mayo 2018

Aprobado: 27 de junio 2018

Conflictos de interés: Los autores declaran no tener conflictos de interés

Fuentes de financiamiento:

Autofinanciado

Citar como: Caballero Ñopo $P$, Alarcón Villaverde J, Piscoya Sara $J R$, Reyes Puma N, Romero Ruiz S, Obregón Cahuayo C, et al. Duración de la supresión viral en pacientes con VIH en terapia antiretroviral en Perú: análisis de sobrevida acumulada 2004-2012. An Fac med. 2018;79(2):125-30.

DOI: http://dx.doi.org/10.15381/anales. v79i2.14938

\begin{abstract}
Resumen
Introducción. El objetivo primordial de los programas nacionales es la supresión de la replicación viral hasta niveles indetectables y el sostenimiento de esta respuesta virológica a lo largo del tiempo. El objetivo del estudio fue analizar la duración de la supresión viral en pacientes adultos con VIH que iniciaron tratamiento antiretroviral en el Perú durante el periodo 2004-2006 con seguimiento hasta el 2012 mediante análisis de sobrevida acumulada. Métodos. Se realizó un estudio observacional retrospectivo realizado en base a la cohorte histórica de pacientes que accedieron al programa nacional de tratamiento antirretroviral durante el periodo 2004 al 2006 en el Perú y que tuvo seguimiento hasta el 31 de diciembre del 2012. Resultados. Del total de 6289 pacientes, 5142(81,7\%) iniciaron algún esquema de tratamiento y 4530(72\%) alcanzaron supresión viral, la cual se sostuvo por tiempo variable durante el periodo de observación. Según la tasa de sobrevida acumulada el 91,1\% mantuvieron supresión viral hasta 1 año, 84,6\% hasta 2 años, $80,2 \%$ hasta 3 años, $77,1 \%$ hasta 4 años, $74,1 \%$ hasta 5 años y $70,1 \%$ hasta 6 años. Conclusiones. La cohorte peruana que empezó el tratamiento antirretroviral en la etapa inicial de implementación del programa nacional, presentó duración de supresión viral sostenida con tasas comparables o por encima a otros países latinoamericanos. La duración de la supresión viral es indicador longitudinal del desempeño de los programas nacionales y locales, que puede contribuir a estimar la duración del uso de un solo tipo de esquema de terapia antiretroviral y validar adherencia.
\end{abstract}

Palabras clave: VIH; Tratamiento; Perú; Sobrevida

\section{Abstract}

Introduction. The primary objective of national programs is the suppression of viral replication to undetectable levels and the sustaining of this virological response over time. The objective of the study was to analyze the duration of viral suppression in adult patients with HIV who started antiretroviral treatment in Peru during the period 2004-2006 with follow-up until 2012 using cumulative survival rate analysis. Methods. A retrospective observational study was conducted based on the historical cohort of patients who linked to the antiretroviral treatment national program during the period 2004 to 2006 in Peru with follow-up until December 31st, 2012. Results. Of the 6289 patients, $5142(81.7 \%)$ initiated some treatment scheme and $4530(72 \%)$ reached viral suppression which was sustained by variable time during the observation period. According to the cumulative survival rate $91.1 \%$ sustained viral suppression up to 1 year, $84.6 \%$ up to 2 years, $80.2 \%$ up to 3 years, $77.1 \%$ up to 4 years, $74.1 \%$ up to 5 years and $70.1 \%$ up to 6 years. Conclusions. The Peruvian cohort that began antiretroviral treatment in the initial phase of implementation of the national program, presented comparable results or above other Latin American countries in the duration of sustained viral suppression. The duration of viral suppression is a longitudinal indicator of the performance of national and local programs, which could also help to estimate the time of use of the therapeutic schemes of antiretrovirals and validate adherence.

Keywords: HIV; Therapeutics; Peru; Survival 


\section{INTRODUCCIÓN}

La infección por el virus de inmunodeficiencia humana (VIH) y el síndrome de inmunodeficiencia adquirida, son en la actualidad controlables con la administración del tratamiento antirretroviral, el cual continuará durante toda la vida de las personas que viven con el $\mathrm{VIH}$. Por ello, el objetivo primordial de los programas nacionales de tratamiento es la supresión de la replicación viral hasta niveles indetectables y el sostenimiento de esta respuesta virológica a lo largo del tiempo. Al presentarse supresión viral sostenida, se mejoran las condiciones clínicas y existe protección contra las infecciones oportunistas que pueden causar complicaciones, se favorece la reconstitución inmune, disminuye la transmisión, morbilidad y mortalidad, y se minimiza el riesgo de emergencia de resistencia a las drogas antirretrovirales ${ }^{1,2,3,4}$.

La mayoría de guías de práctica clínica recomiendan su medición cada seis a doce meses para hacer el seguimiento y detectar los casos de rebote viral o fracaso virológico tempranamente. Esto incluye estrategias para que los pacientes en tratamiento alcancen la supresión viral y la sostengan en el tiempo ${ }^{3,5}$.

En el Perú, en el año 2004, se inició la implementación de un programa de tratamiento con el modelo de sistema desconcentrado, de acceso universal, dirigido desde Lima hacia las provincias que incluía servicios de salud públicos y algunas organizaciones sin fines de lucro acreditadas. Una evaluación realizada luego de los dos primeros años de implementación de este programa demostró la disminución en el número de muertes por SIDA en tanto que se extendía la cobertura del tratamiento. Asimismo, los resultados demostraron que en este periodo inicial, el $76 \%$ de los pacientes alcanzaron la supresión viral (SV) a niveles indetectables (<400 copias virales $/ \mathrm{mm}^{3}$ ) a los 6 meses de tratamiento, observándose además la disminución del número de muertes por SIDA ${ }^{6}$. A nivel mundial, varios estudios demostraron que la mayoría de los pacientes que iniciaron tratamiento antirretroviral, alcanzaron supresión viral a los seis o doce meses. Sin embargo, esta respuesta virológica alcanzada puede no ser permanente o sostenida en el tiempo, con interrupciones expresadas como elevaciones transitorias o repetidas de la carga viral, lo que puede afectar la salud de los pacientes.
Además, la información sobre la duración de la presencia de supresión viral en las cohortes de pacientes en tratamiento es escasamente estudiada. Son actualmente más frecuentes las publicaciones sobre los indicadores de la cascada del continuo del tratamiento, los cuales son de tipo transversal y miden, por ejemplo, la proporción de personas que viven con VIH que reciben tratamiento en servicios de salud y alcanzaron supresión viral; sin embargo, estos podrían tener limitaciones para reflejar la real eficacia de los programas de tratamiento a nivel operativo ${ }^{7,8,9}$.

En el presente estudio examinamos las características de la cohorte de pacientes del programa nacional de quienes iniciaron tratamiento en el periodo 2004 al 2006 alcanzando supresión viral, y la probabilidad de la duración de esta respuesta virológica a lo largo del tiempo.

\section{MÉTODOS}

Se realizó un estudio observacional retrospectivo en base a la cohorte histórica de pacientes que accedieron al programa nacional de tratamiento antirretroviral durante el periodo 2004 al 2006 en el Perú y que tuvo seguimiento hasta el 31 de diciembre del 2012, siendo denominada la cohorte del estudio. Se utilizaron fuentes secundarias de datos con los registros rutinarios del seguimiento y controles de carga viral y CD4 del programa nacional y sistema NETLAB del Instituto Nacional de Salud del Ministerio de Salud del Perú. Los individuos fueron elegidos para inclusión en el estudio si cumplieron con los siguientes criterios: adultos de 18 o más años de edad, de ambos sexos, con diagnostico confirmado de infección por $\mathrm{VIH}$, que accedieron al programa nacional entre el año 2004 y el 2006, en cualquier ciudad del país, que tuvieron controles de seguimiento de pruebas de laboratorio para monitoreo registrados en las bases de datos nacionales del Ministerio de Salud y con dos o más mediciones registradas de carga viral durante el periodo de observación. No se hizo diferencia entre pacientes con o sin antecedentes de tratamiento previo o sobre el tipo de esquema de medicamentos recibidos. Se consideró la fecha de medición basal de CD4 como el momento de acceso del paciente al programa. $Y$ la fecha del primer control de carga viral como el inicio del tratamiento. Se definió la variable "supresión viral sostenida" como la presencia de uno o más controles de carga viral con valores me- nores a 400 copias virales $/ \mathrm{mm}^{3}$ en pacientes bajo tratamiento. Se tomó como referencia el valor <400 copias/ml para definir supresión viral (indetectable), por concordar con las guías clínicas nacionales e internacionales vigentes al inicio del programa (durante el periodo consignado en el estudio) y por ser el límite de detección inferior de la metodología utilizada durante ese periodo.

Se estudiaron además las variables demográficas edad al inicio del tratamiento, sexo, lugar de residencia, establecimiento de salud, año de inicio de tratamiento, valor del CD4 basal, valor de la carga viral basal. La edad fue definida como la diferencia en años cumplidos entre la fecha de nacimiento y la fecha de control basal de CD4 como el momento de acceso al programa. El lugar de residencia para propósito del estudio fue registrado como los lugares o zonas en donde los pacientes inician su tratamiento, pues allí está ubicado el establecimiento de salud. En el caso de Lima, se clasificó en Lima Metropolitana y Lima Provincias. El Callao es una provincia constitucional y no se encuentra dentro de Lima. Todas las otras regiones fueron clasificadas para el estudio en tres grupos: resto de costa, selva y sierra. En la zona denominada "Resto de Costa" se consideró las regiones de Tumbes, Piura, Lambayeque, La Libertad, Ancash, Ica, Arequipa, Moquegua y Tacna; en la zona "Selva" se consideró las regiones Loreto, Amazonas, San Martín, Madre de Dios y Ucayali; y en la zona "Sierra" se consideró las regiones Cajamarca, Pasco, Huánuco, Junín, Huancavelica, Ayacucho, Apurímac, Cusco y Puno.

El establecimiento de salud inicial fue aquel a través del cual el paciente accedió al programa de tratamiento. Se consideró el CD4 basal a la primera medición de la cantidad de linfocitos T-CD4 presentes en sangre periférica del paciente con VIH, basándose los resultados de citometría de flujo; y la carga viral basal como la primera medición de la cantidad de copias de ARN viral en plasma sanguíneo basada en la reacción en cadena de la poliemrasa (PCR) en los pacientes con $\mathrm{VIH}$. En ambos casos se tomaron en cuenta las pruebas de laboratorio realizadas hasta seis meses antes del inicio del tratamiento. Los resultados de controles periódicos de carga viral y CD4 fueron analizados a lo largo del periodo de observación del estudio.

El protocolo del estudio fue evaluado metodológicamente de modo integral y autorizado por la Unidad de Investigación de la Facultad de Medicina y el Vicerrectorado de Investigación de la Universidad Nacional Mayor de San Marcos. 
Para el análisis de datos, se realizó en primer lugar el análisis descriptivo de los datos demográficos y de las mediciones basales de los pacientes con y sin supresión viral a través de la distribución de frecuencias y porcentajes. Se analizó la presencia de supresión viral sostenida a lo largo del tiempo para determinar la proporción acumulada de pacientes que continúan con valores menores a 400 copias virales $/ \mathrm{ml}$, para determinar la sobrevida acumulada según intervalos anuales. Para ello se elaboró la tabla de vida (Life table). Se utilizó el Software libre R, versión 3.0.3.

\section{RESULTADOS}

Se identificaron 6289 pacientes adultos con diagnóstico de infección por VIH - SIDA que tuvieron acceso a evaluación basal en el programa de tratamiento de acceso gratuito conducido por el Ministerio de Salud del Perú y cuyas características se pueden apreciar en la tabla 1 , según los datos registrados en el sistema fuente del estudio. En este grupo de pacientes, la relación hombre-mujer fue de 2:1, la media de la edad 34,19 (DE 9,54) y

Tabla 1. Características de los pacientes que accedieron al programa $2004-2006,(n=6289)$

\section{Variable}

Pacientes $(n=6289)$

Sexo

$\mathrm{F}$

M

Edad

media (DE)
mediana (IQR)
$18-29$
$30-39$
$40-49$
$>=50$

Lugar de residencia

Lima metropolitana
Lima provincias
Callao
Resto de Costa
Selva
Sierra

Año de acceso al programa

2004

2005

2006

Establecimiento de salud

$$
\begin{aligned}
& \text { Hospital público } \\
& \text { Hospital militar } \\
& \text { Institución privada } \\
& \text { Servicios de salud } \\
& \text { media (DE) } \\
& \text { mediana (IQR) } \\
& <3 \\
& 03-\text { may } \\
& >5
\end{aligned}
$$$$
\text { Servicios de salud en cárceles }
$$

Carga viral basal (logaritmo 10)

2080

4209

34,19

33

2272

2453

1075

489

4209

CD4 basal

media (SD)
mediana (IQR)
$<200$
$200-350$
$>350$

(33,1\%) $(66,9 \%)$

(32,6\%)

$(46,1 \%)$

(84,3\%)

$(2,6-8,7)$

$(18,9 \%)$

$(39,1 \%)$

$(42,0 \%)$ $(20,1 \%)$
Fuente: NetLab, Instituto Nacional de Salud, Ministerio de Salud del Perú. la mediana 33 (IQR 18-77). La mayoría de pacientes tuvieron acceso al programa en ciudades de la costa, principalmente Lima $(66,9 \%)$ y el resto de costa $(14,1 \%)$, a través de establecimientos de salud públicos. La media de la carga viral basal fue 5,5 logaritmo $(D E 6,8)$ y una mediana de 4,8 (IQR 2,6-8,7). La media del CD4 basal fue 216,6 (DE 190,9) y una mediana de 175 (IQR 1-1619). En relación a las mediciones basales, la mayoría de los pacientes $(55,3 \%)$ accedieron al programa con un valor basal de CD4 $<200$ células $/ \mathrm{mm}^{3}$ y el $42 \%$ lo hizo con $>5$ logaritmo de carga viral basal.

Del total de 6289 pacientes, 5142 $(81,7 \%)$ iniciaron algún esquema de tratamiento, de los cuales 4530 (72\%) alcanzaron supresión viral que se sostuvo por tiempo variable durante el periodo de observación y 612 pacientes nunca alcanzaron supresión viral. En las tablas 2, 3 y 4 se presenta la distribución porcentual de las características de los pacientes en tratamiento ( $n=5142)$ de acuerdo a las variables demográficas basales, diferenciando los dos grupos con y sin supresión viral. La mayoría de pacientes iniciaron tratamiento con valores basales de carga viral $>5$ logaritmo y valores de CD4 $<200$ células $/ \mathrm{mm}^{3}$.

En la tabla 5 se muestra la tasa de sobrevida acumulada con supresión viral; es decir, la proporción de pacientes que "sobrevivieron" manteniendo supresión viral desde el inicio hasta el final de cada intervalo anual correspondiente al periodo de seguimiento. De acuerdo a los resultados, se puede interpretar que de los 4530 pacientes que alcanzaron supresión viral el 98,2\% continuó con supresión viral durante medio año (primer intervalo), de los 4194 pacientes que sobrevivieron hasta el inicio del siguiente intervalo el $91,1 \%$ continuó con supresión viral hasta el final del año 1 (segundo intervalo); de los 3719 que sobrevivieron hasta el inicio del siguiente intervalo el $84,6 \%$ continuó con supresión viral hasta el final del año 2 (tercer intervalo); de los 3256 que sobrevivieron hasta el inicio del siguiente intervalo el $80,2 \%$ continuó con supresión viral hasta el final del año 3 (cuarto intervalo); de los 2901 que sobrevivieron hasta el 
Tabla 2. Características demográficas basales de los pacientes en tratamiento $(n=5142)$

\begin{tabular}{|c|c|c|c|c|c|c|}
\hline \multirow[t]{2}{*}{ Variable } & & \multicolumn{2}{|c|}{ Con supresión viral $(n=4530)$} & \multicolumn{2}{|c|}{ Sin supresión viral (n=612) } & \multirow[t]{2}{*}{ Total } \\
\hline & & $n$ & $\%$ & $\mathrm{n}$ & $\%$ & \\
\hline \multicolumn{7}{|l|}{ Sexo } \\
\hline & $\mathrm{F}$ & 1550 & 87,5 & 221 & 12,5 & 1771 \\
\hline & M & 2980 & 88,4 & 391 & 11,6 & 3371 \\
\hline \multicolumn{7}{|l|}{ Edad } \\
\hline & $18-29$ & 1529 & 83,3 & 307 & 16,7 & 1836 \\
\hline & $30-39$ & 1837 & 90,0 & 205 & 10,0 & 2042 \\
\hline & $40-49$ & 787 & 90,8 & 80 & 9,2 & 867 \\
\hline & $\geq 50$ & 377 & 93,0 & 20 & 5,0 & 397 \\
\hline \multicolumn{7}{|c|}{ Año de Inicio } \\
\hline & 2004 & 1059 & 94,5 & 62 & 5,5 & 1121 \\
\hline & 2005 & 1454 & 86,9 & 219 & 13,1 & 1673 \\
\hline & 2006 & 2017 & 85,9 & 331 & 14,1 & 2348 \\
\hline \multicolumn{7}{|c|}{ Establecimiento } \\
\hline & Hospital público & 3818 & 88,9 & 476 & 11,1 & 4294 \\
\hline & Hospital militar & 53 & 93,0 & 4 & 7,0 & 57 \\
\hline & Institución privada & 613 & 86,0 & 100 & 14,0 & 713 \\
\hline & Servicios de salud en cárceles & 46 & 59,0 & 32 & 41,0 & 78 \\
\hline
\end{tabular}

Fuente: NetLab, Instituto Nacional de Salud, Ministerio de Salud del Perú.

inicio del siguiente intervalo el $77,1 \%$ continuo con supresión viral hasta el final del año 4 (quinto intervalo); de los 2506 que sobrevivieron hasta el inicio del siguiente intervalo el $74,1 \%$ continuo con supresión viral hasta el final del año 5 (sexto interva- lo); y de los 1969 que sobrevivieron hasta el inicio del siguiente intervalo el 70,1\% continuo con supresión viral hasta el final del año 6 (séptimo intervalo) del periodo de observación del estudio.

\section{DISCUSIÓN}

Nuestros resultados demuestran que la cohorte de nuestros pacientes tiene características similares con países vecinos en relación a la mediana de la edad

Tabla 3. Características demográficas del lugar de residencia de los pacientes en tratamiento $(n=5142)$

\begin{tabular}{|c|c|c|c|c|c|c|}
\hline \multirow{2}{*}{\multicolumn{2}{|c|}{ Lugar de Residencia }} & \multicolumn{2}{|c|}{ Con supresión viral ( $n=4530)$} & \multicolumn{2}{|c|}{ Sin supresión viral ( $n=612$ ) } & \multirow{2}{*}{ Total } \\
\hline & & n & $\%$ & n & $\%$ & \\
\hline \multirow[t]{2}{*}{ Lima } & Lima metropolitana & 3186 & 89,6 & 370 & 10,4 & 3556 \\
\hline & Lima provincias & 61 & 93,8 & 4 & 6,2 & 65 \\
\hline Callao & Callao & 429 & 89,2 & 52 & 10,8 & 481 \\
\hline \multirow{9}{*}{ Resto de la costa } & Ancash & 67 & 80,7 & 16 & 19,3 & 83 \\
\hline & Arequipa & 102 & 82,9 & 21 & 17,1 & 123 \\
\hline & Ica & 61 & 79,2 & 16 & 20,8 & 77 \\
\hline & La Libertad & 79 & 92,9 & 6 & 7,1 & 85 \\
\hline & Lambayeque & 62 & 73,8 & 22 & 26,2 & 84 \\
\hline & Moquegua & 11 & 73,3 & 4 & 36,7 & 15 \\
\hline & Piura & 87 & 85,3 & 15 & 14,7 & 102 \\
\hline & Tacna & 12 & 54,5 & 10 & 45,5 & 22 \\
\hline & Tumbes & 48 & 87,3 & 7 & 12,7 & 55 \\
\hline \multirow[t]{5}{*}{ Selva } & Amazonas & 7 & 70,0 & 3 & 30,0 & 10 \\
\hline & Loreto & 125 & 81,7 & 28 & 18,3 & 153 \\
\hline & Madre de Dios & 18 & 72,0 & 7 & 28,0 & 25 \\
\hline & San Martín & 17 & 73,9 & 6 & 26,1 & 23 \\
\hline & Ucayali & 32 & 97,0 & 1 & 3,0 & 33 \\
\hline \multirow[t]{9}{*}{ Sierra } & Apurímac & 3 & 60,0 & 2 & 40,0 & 5 \\
\hline & Ayacucho & 8 & 100,0 & 0 & 0,0 & 8 \\
\hline & Cajamarca & 6 & 54,5 & 5 & 45,5 & 11 \\
\hline & Cusco & 19 & 95,0 & 1 & 5,0 & 20 \\
\hline & Huancavelica & 4 & 66,7 & 2 & 33,3 & 6 \\
\hline & Huánuco & 19 & 90,5 & 2 & 9,5 & 21 \\
\hline & Junín & 60 & 85,7 & 10 & 14,3 & 70 \\
\hline & Pasco & 4 & 80,0 & 1 & 20,0 & 5 \\
\hline & Puno & 3 & 75,0 & 1 & 25,0 & 4 \\
\hline
\end{tabular}

Fuente: NetLab, Instituto Nacional de Salud, Ministerio de Salud del Perú. 
Tabla 4. Características de marcadores basales de laboratorio en los pacientes en tratamiento $(n=5142)$

\begin{tabular}{llccccc} 
& & $\begin{array}{c}\text { Con supresion viral } \\
\text { (4530) }\end{array}$ & $\begin{array}{c}\text { Sin supresion viral } \\
\text { (612) }\end{array}$ & Total \\
& & $\mathbf{n}$ & $\mathbf{\%}$ & $\mathbf{n}$ & $\mathbf{\%}$ & \\
\hline Carga viral basal (Log) & & & & & & \\
& $<3$ & 1018 & 96,2 & 40 & 3,8 & 1058 \\
& 3 a 5 & 1640 & 80,9 & 388 & 19,1 & 2028 \\
& $>5$ & 1872 & 91,1 & 184 & 8,9 & 2056 \\
& & & & & & \\
& $<200$ & 2559 & 94,3 & 156 & 5,7 & 2715 \\
& $200-350$ & 1208 & 88,4 & 159 & 11,6 & 1367 \\
& $>350$ & 763 & 72,0 & 297 & 28,0 & 1060 \\
\hline
\end{tabular}

Fuente: NetLab, Instituto Nacional de Salud, Ministerio de Salud del Perú al inicio del tratamiento y la mediana del valor basal de CD4. Esta cohorte inicial de pacientes peruanos que ingresó al tratamiento antirretroviral en condiciones de larga espera sin tratamiento y con deterioro inmunológico ha presentado una evolución virológica aceptable, representada por un $72 \%$ de pacientes que alcanzan supresión viral y por la tasa de sostenimiento de esta supresión que a lo largo de 6 años ha sido del 71,1\%; teniendo en consideración que el estudio está limitado a medir como evento, únicamente el primer episodio de interrupción de supresión viral.

La razón de pacientes con supresión viral / sin supresión viral fue 7,4; sin embargo, se ha encontrado que este resultado global no refleja la realidad nacional y está sesgado por la elevada cantidad de pacientes que reciben tratamiento en Lima y Callao, en donde existen servicios de salud con mayor experiencia y recursos, mientras que en siete regiones el porcentaje de pacientes con supresión viral estuvo por debajo del $50 \%$.
Estos procesos de implementación y desconcentración de los servicios de salud para brindar tratamiento a pacientes con VIH han sucedido también en otros países de Latinoamérica. Todos tienen en común ser estudios retrospectivos en los que se utilizó los datos de historias clínicas o de sistemas rutinarios de información que contienen datos demográficos básicos, fechas de controles, resultados de controles y en algunos casos registro del tipo de medicamentos antiretrovirales administrados a los pacientes. Por lo tanto, no existe en la actualidad uniformidad o criterios para medir el sostenimiento de la supresión viral en el tiempo, como un indicador longitudinal de evaluación del programa. Ante ello, en todos los estudios, un resultado a evaluar ha sido la falla virológica con definiciones operacionales diferentes, por lo que esto debe tenerse en cuenta al realizar comparaciones. En el estudio realizado en Santos, Brasil, Caseiro et. al. analizó los pacientes que han presentado respuesta exitosa de supresión viral por 48 semanas y que fueron seguidos para identificar
Tabla 5. Resultados del análisis de sobrevida acumulada de la supresión viral $(n=4530)$

\begin{tabular}{lccccc}
$\begin{array}{l}\text { Intervalo } \\
\text { anual }\end{array}$ & $\begin{array}{c}\mathbf{N}^{\circ} \text { de pacientes } \\
\text { con SV al inicio del } \\
\text { intervalo }\end{array}$ & $\begin{array}{c}\mathbf{N}^{\circ} \text { de pacientes } \\
\text { que salieron de } \\
\text { la SV }\end{array}$ & Pérdidas & $\begin{array}{c}\mathbf{N}^{\circ} \text { de } \\
\text { expuestos a } \\
\text { riesgo }\end{array}$ & $\begin{array}{c}\text { Sobrevida } \\
\text { acumulada }\end{array}$ \\
\hline 0,5 & 4530 & 80 & 256 & 4402 & 0,982 \\
1 & 4194 & 297 & 178 & 4105 & 0,911 \\
2 & 3719 & 257 & 206 & 3616 & 0,846 \\
3 & 3256 & 165 & 190 & 3161 & 0,802 \\
4 & 2901 & 105 & 290 & 2756 & 0,771 \\
5 & 2506 & 91 & 456 & 2283 & 0,741 \\
6 & 1969 & 79 & 956 & 1491 & 0,701 \\
\hline
\end{tabular}

SV=Supresión viral cuando presentan falla virológica y cuáles son sus características, definiendo falla virológica como un resultado de carga viral >500 copias luego de inclusión en el estudio ${ }^{10}$. En Argentina, Socías et. al. realizó un estudio en una cohorte pequeña con menos de 80 pacientes diagnosticados en la era previa a los programas de tratamiento, propuso definirla como un valor de carga viral $>50$ copias luego de 6 meses de tratamiento ${ }^{11}$. Existe también un estudio peruano realizado en un hospital de Lima, en el cual Jorge et. al. evaluó los factores asociados a la respuesta virológica, y definió falla virológica como la presencia de carga viral >1000 copias luego de 24 semanas de tratamiento ${ }^{12}$. El estudio colombiano de Díaz-Granados et. al. tuvo como objetivo evaluar si los pacientes presentaron en la primera carga viral luego de 48 semanas de tratamiento un valor $<400$ copias, de ese modo evaluaron la tasa de optima respuesta y propusieron predictores ${ }^{13}$. El estudio mexicano realizado por CrabtreeRamirez et. al. tuvo como objetivo evaluar la efectividad del programa de tratamiento, definiendo falla virológica como la presencia de carga viral $>500$ copias luego de 6 meses de tratamiento ${ }^{14}$. Por tanto, existe en común la intención de los países vecinos, con los que compartimos características sociales, culturales y demográficas, de evaluar la respuesta virológica en los pacientes de sus programas de tratamiento y el mejor indicador es la supresión viral, la que debe ser sostenida para asegurar el éxito del programa. Cada país ha planteado su propia metodología, pero el fondo es el mismo. Nuestro estudio ha propuesto evaluar la duración de la supresión viral teniendo como punto de partida el momento en que el paciente alcanza un control de carga viral con valor < 400 copias. Luego de este punto, se realizó el seguimiento del paciente en todos los controles sucesivos hasta que presente su primer evento de interrupción, con un incremento de la carga viral a valores $>400$. En nuestro estudio, no hemos definido a la variable principal como "falla virológica" porque las guías clínicas detallan que este término incluye en su definición datos clínicos simultáneos con los que no contamos en las fuentes de bases de datos secundarias analizadas. 
Por otro lado, en relación a los resultados obtenidos, el estudio de Brasil consideró en su cohorte retrospectiva a 4909 pacientes en tratamiento. El 27,5\% (669) de los pacientes alcanzaron supresión viral durante 48 semanas consecutivas y utilizaron el test de Kaplan-Meyer para analizar su duración después de las primeras 48 semanas de buena respuesta. Los resultados demostraron que las mujeres presentaron falla virológica más temprano que los hombres y que no existía correlación entre las mediciones basales de CD4 o carga viral con la duración de la supresión viral ${ }^{10}$. En el estudio mexicano se analizaron los datos en una cohorte retrospectiva de pacientes enrolados del 2001 al 2005, atendida en un centro especializado de México distrito Federal, con el objetivo de evaluar la respuesta virológica, duración y resultados clínicos del primer régimen de tratamiento. Fueron elegibles para el análisis 191/377 pacientes y mediante el test de KaplanMeyer se mostró que la probabilidad de presentar falla virológica a los 4 años de seguimiento fue $<20 \%$ y que la edad $<30$ años fue un factor de riesgo para falla virológica. La población principalmente atendida en este centro eran hombres que adquirieron el virus por contacto homosexual. Este fue el primer estudio que evaluó resultados entre pacientes que iniciaron tratamiento dentro del programa del gobierno ${ }^{14}$.

En nuestro estudio, de modo global el $72 \%$ de pacientes alcanzo supresión viral y en este grupo, los resultados presentados de su duración, están por encima de los de Brasil y Mexico. La cohorte peruana que empezó el tratamiento antirretroviral en la etapa inicial de implementación del programa nacional, presentó una duración de supresión viral sostenida con tasas comparables o por encima a otros países latinoamericanos.

Al igual que en los inicios, donde fue importante promover el acceso de las personas a los programas de tratamiento, ahora es necesario evaluar de modo estandarizado y representativo el grado de alcance de la supresión viral y la presencia de fármaco resistencia entre los pacientes que fracasan al tratamiento. Por ello, la OMS estableció desde el año 2013 las recomendaciones de la medición de prevalencia de supresión viral en los pacientes que recibieron tratamiento a los 12 (etapa temprana de exposición al tratamiento) y 48 (etapa tardía de exposición al tratamiento) meses. El resultado de esta medición puede proveer información valiosa acerca del desempeño de los programas nacionales de los países ${ }^{3}$.

Se tuvo como principal limitación el tiempo prolongado empleado en la elaboración de la base de datos del estudio, que tuvo como fuente el sistema de reporte de resultados de laboratorio.

La principal contribución del estudio es proveer evidencia de la utilidad de medir la duración de la supresión viral como un indicador longitudinal de respuesta virológica para evaluar el desempeño de los programas. La duración de la supresión viral como indicador proxy puede contribuir a estimar la duración del uso de un mismo tipo de esquema de terapia antiretroviral y además validar la presencia de adherencia. Un aporte adicional del estudio es el conocimiento de las diferencias regionales de la respuesta virológica en el Perú.

\section{AGRADECIMIENTOS}

La presente investigación se realizó gracias al trabajo conjunto de investigadores del Grupo de Investigación de Infecciones de Transmisión Sexual del Instituto de Medicina Tropical Daniel A. Carrión de la Universidad Nacional Mayor de San Marcos y del Instituto Nacional de Salud.

\section{REFERENCIAS BIBLIOGRÁFICAS}

1. European AIDS Clinical Society Guidelines, Versión 8.2. January 2017. Disponible en: http://www. eacsociety.org/files/guidelines_8.2-english.pdf [Acceso 14 de junio de 2018].

2. Panel on Antiretroviral Guidelines for Adult and Adolescents. Guidelines for the use of antiretroviral agents in HIV-1 Infected adults and adolescents living with HIV. Department of Health and Human Services. 2018. Disponible en: https://aidsinfo.nih. gov/contentfiles/Ivguidelines/adultandadolescentgl.pdf [Acceso 15 de junio de 2018].

3. World Health Organization. Consolidated Guidelines on the use of antiretroviral drugs for treating and preventing HIV infection: recommendations for a public health approach - second edition. 2016. Disponible en: http://apps.who.int/iris/bitstream/ handle/10665/208825/9789241549684_eng.pdf; jsessionid $=3763$ F60AED647A6B5544A87D3B08 5C2D?sequence $=1$ [Acceso 10 de mayo de 2018].
4. Aberg JA, Gallant JE, Ghanem KG, Emmanuel P, Zingman BS, Horberg MA. Infectious Diseases Society of North America. Primary Care Guidelines for the Management of Persons Infected With HIV: 2013 Update by the HIV Medicine Association of the Infectious Diseases Society of America. Clinical Infectious Diseases. 2014 Jan;58(1):1-10. DOI: 10.1093/cid/cit757.

5. Ministerio de Salud. Norma Técnica de Salud de Atención Integral del Adulto con Infección por el VIH. Resolución Ministerial N²15-2018/MINSA. 2018. Disponible en: http://busquedas.elperuano. pe/normaslegales/aprueban-la-nts-n-097-minsadgsp-v03-norma-tecnica-de-sal-resolucionministerial-no-215-2018minsa-1626125-2/ [Acceso 10 de mayo de 2018].

6. Sebastián Mesones JL, Caballero Ñopo P, Sánchez Fernández J, Grant R, Podestá Gavilano L E. A step forward in the fight against AIDS. The first two years of universal access of antiretroviral treatment in Peru. ( $1^{\circ}$ edición.). Lima, Perú: 2006. Disponible en: ftp:// ftp2.minsa.gob.pe/descargas/03esn/03itssida/ ATRetroviral/ATR_PreliminaryPages.pdf [Acceso 15 de mayo de 2018].

7. McMahon $\mathrm{JH}$, Elliott $\mathrm{JH}$, Bertagnolio $\mathrm{S}$, Kubiak $\mathrm{R}$, Jordan MR. Viral suppression after 12 months of antiretroviral therapy in low- and middle-income countries: a systematic review. Bull World Health Organ. 2013;91:377-385. DOI: http://dx.doi.org/10.2471/ BLT.12.112946

8. Wolff MJ, Giganti MJ, Cortes CP, Cahn P, Grinsztejn B, Pape JW, et al. A decade of HAART in Latin America: Long term outcomes among the first wave of HIV patients to receive combination therapy. PLoS ONE. 2017;12(6):e0179769. DOI: https://doi.org/10.1371/ journal.pone.0179769

9. Boender TS, Sigaloff KCE, McMahon JH. Long-term virological outcomes of first-line antiretroviral therapy for HIV-1 in low- and middle-income countries: a systematic review and metaanalysis. Clin Infect Dis. 2015;61(9):1453-1461. DOI:10.1093/cid/civ556

10. Caseiro MM, Golegã AAC, Etzel A, Diaz RS. Characterization of virologic failure after an initially successful 48-week course of antiretroviral therapy in HIV/AIDS outpatients treated in Santos, Brazil. Brazilian Journal of Infectious Diseases. 2008;12(3):162166. DOI:10.1590/S1413-86702008000300001

11. Socias ME, Rotryng F, Lapadula P, Medrano M, Paz D, Stern L, Pryluka D. Treatment as prevention in resource-limited settings: is it feasible to maintain HIV viral load suppression over time?. The Journal of Infection in Developing Countries. 2013;7(08):593599. DOI:10.3855/jidc.2926

12. Jorge AR, Jorge PB, Elsa GL, Miguel CS, Rodriguez M, Willig J, Juan EZ. Risk factors associated with virologic failure in HIV-infected patients receiving antiretroviral therapy at a public hospital in Peru. Revista chilena de infectologia. 2013;30(1):42-48. DOI:10.4067/S0716-10182013000100006

13. Diaz Granados CA, Silva A, Bermudez A, Roncancio $D$, Diruggiero P, Mantilla M. Rate and predictors of optimal virologic response to antiretroviral therapy in Colombia. International Journal of Infectious Diseases. 2007;11(6):531-535. DOI:10.1016/j. ijid.2007.03.002

14. Crabtree-Ramirez B, Villasis-Keever A, GalindoFraga A, del Rio C, Sierra-Madero J. Effectiveness of Highly Active Antiretroviral Therapy (HAART) Among HIV-Infected Patients in Mexico. AIDS Research and Human Retroviruses. 2010; 26(4): 373-378. DOI:10.1089/aid.2009.0077 